\title{
On Irregularities of Bidegreed Graphs
}

\author{
Tamás Réti ${ }^{1}$, Darko Dimitrov ${ }^{2}$ \\ ${ }^{1}$ Óbuda University \\ Bécsi út 96/B, H-1034 Budapest, Hungary \\ E-mail: reti.tamas@bgk. uni-obuda.hu \\ ${ }^{2}$ Institut für Informatik, Freie Universität Berlin \\ Takustraße 9, D-14195 Berlin, Germany \\ \& Hochschule für Technik und Wirtschaft Berlin \\ Wilhelminenhofstraße 75A, D-12459 Berlin, Germany \\ E-mail: dimdar@zedat.fu-berlin.de
}

\begin{abstract}
A graph is regular if all its vertices have the same degree. Otherwise a graph is irregular. To measure how irregular a graph is, several graph topological indices were proposed including: the Collatz-Sinogowitz index [8], the variance of the vertex degrees [7], the irregularity of a graph [4], and recently proposed the total irregularity of a graph [1]. Here, we compare the above mentioned irregularity measures for bidegreed graphs.
\end{abstract}

Keywords: topological graph indices; complete split graph; 2-walk linear graph

\section{Introduction}

All graphs considered here are simple and undirected. Let $G$ be a graph of order $n=|V(G)|$ and size $m=|E(G)|$. For $v \in V(G)$, the degree of $v$, denoted by $d_{G}(v)$, is the number of edges incident to $v$. The adjacency matrix $A(G)$ of a graph $G$ is a matrix with rows and columns labeled by graph vertices, with a 1 or a 0 in position $\left(v_{i}, v_{j}\right)$ according to whether vertices $v_{i}$ and $v_{j}$ are adjacent or not. The characteristic polynomial $\phi(G, t)$ of $\mathrm{G}$ is defined as characteristic polynomial of $A(G): \phi(G, \lambda)=\operatorname{det}\left(\lambda \mathbf{I}_{\mathbf{n}}-A(G)\right)$, where $\mathbf{I}_{\mathbf{n}}$ is $n \times n$ identity matrix. The set of eigenvalues of the adjacent matrix $A(G)$ of a graph $G$ is called a graph spectrum. The largest eigenvalue of $A(G)$, denoted by $\rho(G)$, is called the spectral radius of $G$. An eigenvalue of a graph $\mathrm{G}$ is called main eigenvalue if it has an eigenvector the sum of whose entries is not equal to zero.

In the sequel, we present the irregularity measures consider in this paper. Collatz-Sinogowitz [8] introduced the irregularity measure of a graph $G$ as

$$
\mathrm{CS}(G)=\rho(G)-\frac{2 m}{n} .
$$


An alternative to $\operatorname{CS}(G)$ is the variance of the vertex degrees

$$
\operatorname{Var}(G)=\frac{1}{n} \sum_{i=1}^{n} d_{G}^{2}\left(v_{i}\right)-\frac{1}{n^{2}}\left(\sum_{i=1}^{n} d_{G}\left(v_{i}\right)\right)^{2} .
$$

Bell [7] was first who has compared $\operatorname{CS}(G)$ and $\operatorname{Var}(\mathrm{G})$ and showed that they are not always compatible. Albertson [4] defines the imbalance of an edge $e=u v \in E$ as $\left|d_{G}(u)-d_{G}(v)\right|$ and the irregularity of $G$ as

$$
\operatorname{irr}(G)=\sum_{u v \in E}\left|d_{G}(u)-d_{G}(v)\right|
$$

Recently, in [1] a new measure of irregularity of a simple, undirected graph, so-called the total irregularity, was defined as

$$
\operatorname{irr}_{t}(G)=\frac{1}{2} \sum_{u, v \in V(G)}\left|d_{G}(u)-d_{G}(v)\right| .
$$

More about the above presented irregularity measures, comparison studies of them, and other attempts to measure the irregularity of a graph, one can find in $[3,6,10-12]$. It is interesting that the above four irregularity measures are not always compatible for some pairs of graphs. In this paper we study the relations between the above mentioned irregularity measures for bidegreed graphs.

A universal vertex is the vertex adjacent to all other vertices. A set of vertices is said to be independent when the vertices are pairwise non-adjacent. The vertices from an independent set are independent vertices.

The degree set, denoted by $\mathcal{D}(G)$, of a simple graph $G$ is the set consisting of the distinct degrees of vertices in $G$.

The distance between two vertices in a graph is the number of edges in a shortest path connecting them. The eccentricity of a vertex $v$ in a connected graph $G$ is the maximum graph distance between $v$ and any other vertex of $G$. The radius of a graph $G$, denoted by $\operatorname{rad}(G)$, is the minimum graph eccentricity of any graph vertex of $G$. The diameter of a graph $G$, denoted by $\operatorname{diam}(G)$, is the maximal graph eccentricity of any graph vertex of $G$.

Let $m_{r, s}$ denotes the number of edges in $G$ with end-vertex degrees $r$ and $s$, and let $n_{r}$ denotes the numbers of vertices n $G$ with degree $r$. Numbers $m_{r, s}$ and $n_{r}$ are referred as the edge-parameters and the vertex-parameters of $G$, respectively . The mean degree of a graph $G$ is defined as $\bar{d}(G)=2 m / n$. Graphs $G_{1}$ and $G_{2}$ are said to be edge-equivalent if for their corresponding edge-parameters sets $\left\{m_{r, s}\left(G_{1}\right)>0\right\}=\left\{m_{r, s}\left(G_{2}\right)>0\right\}$ holds. Analogously, they are called vertex-equivalent if for their vertex-parameters sets $\left\{n_{r}\left(G_{1}\right)>\right.$ $0\}=\left\{n_{r}\left(G_{2}\right)>0\right\}$ is fulfilled. It is easy to see that if two graphs are edgeequivalent, then they are vertex-equivalent, as well. 
For two graphs $G_{1}$ and $G_{2}$ with disjoint vertex sets $V\left(G_{1}\right)$ and $V\left(G_{2}\right)$ and disjoint edge sets $E\left(G_{1}\right)$ and $E\left(G_{2}\right)$ the disjoint union of $G_{1}$ and $G_{2}$ is the graph $G=G_{1} \cup G_{2}$ with the vertex set $V\left(G_{1}\right) \cup V\left(G_{2}\right)$ and the edge set $E\left(G_{1}\right) \cup E\left(G_{2}\right)$. The join $G+H$ of simple undirected graphs $G$ and $H$ is the graph with the vertex set $V(G+H)=V(G) \cup V(H)$ and the edge set $E(G+H)=E(G) \cup E(H) \cup\{u v: u \in V(G), v \in V(H)\}$. Let $C_{n}$ denote a cycle on $n$ vertices. Further, let $K_{n}$ denote the complete graph on $n$ vertices, and $t K_{1}$ denote the graph with $t$ isolated vertices and no edges.

A graph $G$ is a complete $k$-partite graph if there is a partiton $V_{1} \cup \cdots \cup V_{k}=$ $V(G)$ of the vertex set, such that $u v \in E(G)$ if and only if $u$ and $v$ are in different parts of the partition. A connected bipartite graph $G$ is semiregular if every edge of $G$ joins a vertex of degree $\delta$ to a vertex of degree $\Delta$. A connected graphs $G$ is called a balanced irregular graph if the equality $\operatorname{irr}(G)=\operatorname{irr}_{t}(G)$ holds.

The rest of the paper is structured as follows. In Section 2 we present some types of bidegreed graphs and some known results related to the above mentioned irregularity measures. In Section 3 we investigate new relations between irregularity indices of bidegreed graphs. Bidegreed graphs with same irregularity indices are investigated in Section 4 . We conclude with final remarks and open problems in Section 5.

\section{Some types of bidegreed graphs and known results}

A graph $G$ is called bidegreed if its degree set $\mathcal{D}(G)=\{\Delta, \delta\}$ with $\Delta>\delta \geq 1$. In the sequel, we present some special types of connected bidegreed graphs that will be of interest later.

i) A bidegreed graph is called a balanced bidegreed graph if the equality $n_{\Delta} n_{\delta}=m_{\Delta, \delta}$ holds for it. It should be noted that the complete bipartite graphs, for which $m=m_{\Delta, \delta}=n_{\Delta} n_{\delta}=\Delta \delta$ holds, form a subset of balanced bidegreed graphs.

ii) A balanced bidegreed graph with $n$ vertices is called a complete split graph if it contains $q=n_{\Delta} \geq 1$ universal vertices and $n-q$ independent vertices [5]. Thus, a complete split graph, denoted by $G_{c s}(n, q)$, can be obtained as join of $n-q$ graphs $K_{1}$ and the complete graph $K_{q}$, i.e., $G_{c s}(n, q)=(n-q) K_{1}+$ $K_{q}$. An existing complete split graph $G_{c s}(n, q)$ is uniquely defined by their parameters $n$ and $q$. This implies that two complete split graphs with identical $n, q$ parameters are isomorphic. For a complete split graph the equalities $m=m_{\Delta, \delta}+m_{\Delta, \Delta}$ and $2 m=(2 n-1) \delta-\delta^{2}$ hold [5].

iii) A balanced bidegreed graph is called a complete split-like graph, denoted by $G_{c s l}(n, q, \delta)$, if it has $q \geq 1$ universal vertices. This implies that for a complete split-like graph the equality $q n_{\delta}=m_{\Delta, \delta}$ holds. The complete split 
graphs represent a subset of complete split-like graphs. It is easy to see that if $\mathrm{G}$ is a complete split-like graph then the equalities $\operatorname{rad}(G)=1$ and $\operatorname{diam}(G)=$ 2 are fulfilled. In Fig. 1 non-isomorphic complete split-like graphs with 5 and 6 vertices are depicted. Note that they are not complete split graphs.

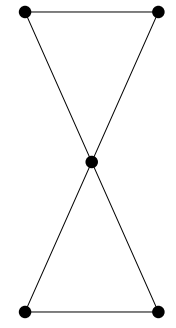

(a)

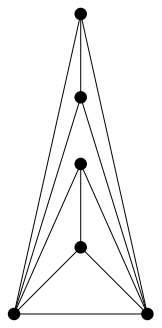

(b)

Fig. 1. Complete split-like graphs $(a) G_{c s l}(5,1,2)$ and $(b) G_{c s l}(6,2,3)$

Also note that since for a complete split-like graph $G q n_{\delta}=m_{\Delta, \delta}$, it follows that if $G$ is not a complete bipartite graph, then $G$ is non-bipartite and contains a triangle.

iv) In a particular case, if $q=1$, then a complete split-like graph is called a generalized windmill graph and is denoted by $G_{c s l}(n, 1, \delta)$. We would like to recall that the classical windmill graph, denoted by $W_{d}(k, p)$, can be constructed by joining $p$ copies of the complete graph $K_{k}$ with a common vertex. For a generalized windmill graph the equality $m=m_{\Delta, \delta}+m_{\delta, \delta}$ is fulfilled. It follows that the star graphs $S_{n}$ with $n \geq 3$ vertices, the wheel graphs $W_{n}$ with $n \geq 5$ vertices, and the classical windmill graphs $W_{d}(k, p)$ with $(k-1) p+1$ vertices and $p k(k-1) / 2$ edges defined for $k \geq 2$ and $p \geq 2$ positive integers, form the subsets of generalized windmill graphs. In Fig. 2 two non-isomorphic generalized windmill graphs are depicted.

Next, we state some known results that will be used afterwords.

Lemma 1 ( [16]). Let $G$ be a connected bidegreed graph with spectral radius $\rho(G)$. Then

$$
\rho(G)=\sqrt{\frac{1}{n} \sum_{u \in V(G)} d^{2}(u)}=\sqrt{\Delta \delta}
$$

if and only if $G$ is a semiregular connected bipartite graph.

Lemma 2 ( $[\mathbf{1 5}])$. Let $G$ be a connected graph with mean degree $\bar{d}(G)]=$ $2 m / n$, and just two main eigenvalues, $\rho$ and $\mu<\rho$, where $\rho$ is the spectral 

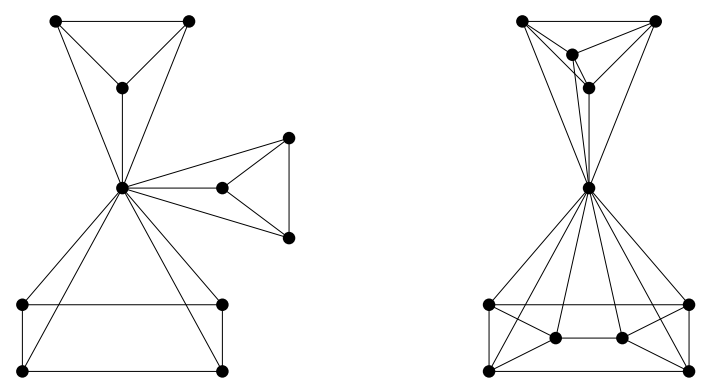

Fig. 2. Two generalized windmill graphs

radius of $G$. Then

$$
\operatorname{Var}(G)=\frac{1}{n} \sum_{u \in V(G)} d^{2}(u)-\left(\frac{2 m}{n}\right)^{2}=\left(\rho-\frac{2 m}{n}\right)\left(\frac{2 m}{n}-\mu\right) .
$$

Lemma 3 ( [15]). Let $G$ be a connected graph with spectral radius $\rho$. Then $G$ is a semiregular bipartite graph if and only if the main eigenvalues of $G$ are $\rho$ and $-\rho$.

Lemma 4 ( [13]). Let $G$ be a connected graph with spectral radius $\rho$. Then

$$
\rho(G) \leq \frac{\delta-1+\sqrt{(\delta+1)^{2}+4(2 m-\delta n)}}{2} .
$$

Equality holds if and only if $G$ is regular or a bidegreed graph in which each vertex is of degree either $\delta$ or $n-1$.

\section{Relations between irregularity indices - new results}

In this section, we present some new results about the relations between irregularity indices of bidegreed graphs. We start with the following simple proposition.

Proposition 1. Let $G(\Delta, \delta)$ be a connected bidegreed graph having $n_{\Delta}$ and $n_{\delta}$ vertices with degree $\Delta$ and $\delta$, respectively. Then the following relations hold:

$$
\begin{aligned}
& m=m(G(\Delta, \delta))=m_{\Delta, \Delta}+m_{\Delta, \delta}+m_{\delta, \delta} \geq m_{\Delta, \delta}, \\
& \operatorname{irr}(G(\Delta, \delta))=m_{\Delta, \delta}(\Delta-\delta), \\
& \operatorname{irr}_{t}(G(\Delta, \delta))=n_{\Delta} n_{\delta}(\Delta-\delta)=n_{\Delta}\left(n-n_{\Delta}\right)(\Delta-\delta), \\
& \operatorname{irr}_{t}(G(\Delta, \delta))=\frac{n_{\Delta} n_{\delta}}{m_{\Delta, \delta}} \operatorname{irr}(G(\Delta, \delta)) .
\end{aligned}
$$


Proof. It is obvious that for a connected bidegreed graph $G(\Delta, \delta)$ the equality $m=m_{\Delta, \delta}$ holds if and only if $G(\Delta, \delta)$ is semiregular. The equalities (6), (7) and (8) follow from the definitions of irregularity indices.

Because the function $f\left(n_{\Delta}\right)=n-n_{\Delta}$ has a maximum value for $n_{\Delta}=n / 2$, we have the following corollary.

Corollary 1. For a connected bidegreed graph $G(\Delta, \delta)$ it holds that

$$
\operatorname{irr}_{t}(G(\Delta, \delta))=n_{\Delta}\left(n-n_{\Delta}\right)(\Delta-\delta) \leq \frac{n^{2}}{4}(\Delta-\delta)
$$

Inequality (9) is sharp. There exist bidegreed graphs with $n$ vertices for which $\operatorname{irr}_{t}(G(\Delta, \delta))=n^{2}(\Delta-\delta) / 4$. Such bidegreed graphs with 8 vertex and deegre set $\{3,4\}$ are shown in Fig. $3(a)$. These graphs are non edge-equivalent, but only vertex equivalent, and the equality $n_{3}=n_{4}=n / 2=4$ holds for them. Another example of bidegreed graphs that satisfy equality in (9) is given in Fig. 3(b). Those graphs are with 8 vertices and have deegre set $\{2,3\}$. They are edge-equivalent, and satisfy the equality $n_{2}=n_{3}=n / 2=4$. It is interesting to note that the graphs in Fig. $3(b)$ are not only edge-equivalent $\left(m_{2,3}=8, m_{3,3}=2\right)$, but they have identical spectral radius $(1+\sqrt{17}) / 2$, as well. Consequently, all considered irregularity indices (CS, Var, irr and irrt ) are identical for them.

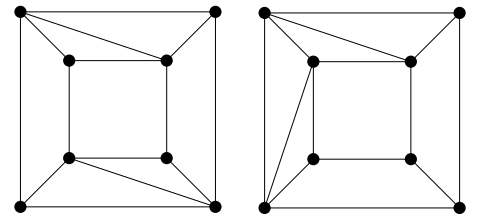

(a)

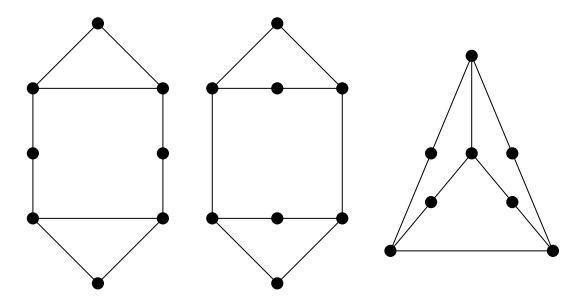

(b)

Fig. 3. Examples of non-isomorphic bidegreed graphs with 8 vertices with identical maximum total irregularity indices

Proposition 2. Let $G(\Delta, \delta)$ be a connected bidegreed graph, then

$$
\operatorname{irr}_{t}(G(\Delta, \delta))=\frac{\Delta-\delta}{\Delta \delta}\left(m^{2}-\left(m_{\Delta, \Delta}-m_{\delta, \delta}\right)^{2}\right) \leq \frac{\Delta-\delta}{\Delta \delta} m^{2}
$$

The equality holds if $m_{\Delta, \Delta}=m_{\delta, \delta}$. 
Proof. For any bidegreed graph $G(\Delta, \delta)$, it holds that

$$
\begin{aligned}
\Delta n_{\Delta} & =m_{\Delta, \delta}+2 m_{\Delta, \Delta}, \quad \text { and } \\
\delta n_{\Delta} & =m_{\Delta, \delta}+2 m_{\delta, \delta} .
\end{aligned}
$$

This together with (7) implies that

$$
\operatorname{irr}_{t}(G(\Delta, \delta))=\frac{\Delta-\delta}{\Delta \delta}\left(m_{\Delta, \delta}+2 m_{\Delta, \Delta}\right)\left(m_{\Delta, \delta}+2 m_{\delta, \delta}\right) .
$$

Since $m_{\Delta, \delta}=m-m_{\Delta, \Delta}-m_{\delta, \delta}$, it follows that

$$
\operatorname{irr}_{t}(G(\Delta, \delta))=\frac{\Delta-\delta}{\Delta \delta}\left(m^{2}-\left(m_{\Delta, \Delta}-m_{\delta, \delta}\right)^{2}\right) \leq \frac{\Delta-\delta}{\Delta \delta} m^{2} .
$$

The equality in (10) is obtained when $m_{\Delta, \Delta}=m_{\delta, \delta}$. This condition holds for the bidegreed graphs with 10 vertices and 12 edges in Fig. 4. Consequently all of them have the same maximum total irregularity index $\operatorname{irr}_{t}=n_{2} n_{3}=$ $6 \cdot 4=24$.

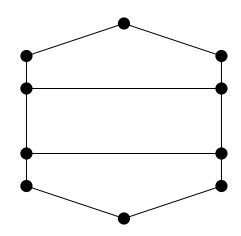

$J_{a}$

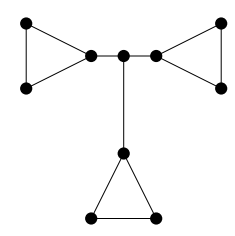

$J_{b}$

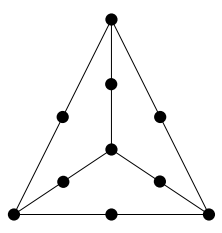

$J_{c}$

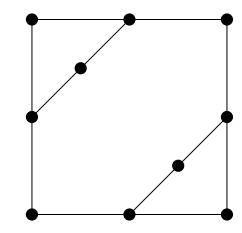

$J_{d}$

Fig. 4. Bidegreed graphs having identical vertex degree set $\left(n_{3}=4, n_{2}=6\right)$ and identical maximum total irregularity index $\operatorname{irr}_{t}=24$

Among bidegreed graphs having identical vertex degree set $\left(n_{\Delta}, n_{\delta}\right)$, the semiregular graphs (for which the equality $m_{\Delta, \Delta}=m_{\delta, \delta}=0$ holds) possess the maximal irregularity $\operatorname{irr}(G)$, as it is a case with graphs $J_{c}$ and $J_{d}$ in Fig. 4.

\section{Bidegreed graphs with same irregularity indices}

In the following we will show that there exists a broad class of bidegreed graphs having "similar irregularity", or in other words, there exist nonisomorphic graph pairs for which two (or more than two) irregularity indices are equal. Moreover, we will show that there are some particular classes of bidegreed graphs whose irregularity indices are considered algebraically dependent quantities. 


\subsection{Balanced bidegreed graphs}

From the definition of balanced bidegreed graphs, it follows that

$$
\operatorname{irr}(G(\Delta, \delta))=\operatorname{irr}_{t}(G(\Delta, \delta))=n_{\Delta} n_{\delta}(\Delta-\delta)=m_{\Delta, \delta}(\Delta-\delta) .
$$

This implies that the balanced bidegreed graphs form a subset of balanced irregular graphs.

Proposition 3. Let $G(\Delta, \delta)$ be a balanced bidegreed graph for which $m_{\Delta, \Delta}=$ 0 or $m_{\delta, \delta}=0$ hold. Then

$$
\operatorname{irr}(G(\Delta, \delta))=\operatorname{irr}_{t}(G(\Delta, \delta))=(2 m-\Delta \delta)(\Delta-\delta)
$$

Proof. For any bidegreed graph $G(\Delta, \delta)$

$$
\begin{gathered}
\Delta n_{\Delta}=m_{\Delta, \delta}+2 m_{\Delta, \Delta}, \\
\delta n_{\delta}=m_{\Delta, \delta}+2 m_{\delta, \delta} .
\end{gathered}
$$

Consequently, we get

$$
\begin{gathered}
n_{\Delta} n_{\delta}=m_{\Delta, \delta}=\frac{\left(m_{\Delta, \delta}+2 m_{\Delta, \Delta}\right)\left(m_{\Delta, \delta}+2 m_{\delta, \delta}\right)}{\Delta \delta}, \quad \text { and } \\
m_{\Delta, \delta}^{2}+\left(2\left(m_{\Delta, \Delta}+m_{\delta, \delta}\right)-\Delta \delta\right) m_{\Delta, \delta}+4 m_{\Delta, \Delta} m_{\delta, \delta}=0 .
\end{gathered}
$$

Taking into consideration that $m_{\Delta, \Delta}+m_{\delta, \delta}=m-m_{\Delta, \delta}$, we have

$$
m_{\Delta, \delta}^{2}+(\Delta \delta-2 m) m_{\Delta, \delta}-4 m_{\Delta, \Delta} m_{\delta, \delta}=0 .
$$

Because $m_{\Delta, \delta}$ is a positive number it is easy to see that the proper solution of the equation above is

$$
n_{\Delta} n_{\delta}=m_{\Delta, \delta}=\frac{1}{2}\left(2 m-\Delta \delta+\sqrt{(2 m-\Delta \delta)^{2}+16 m_{\Delta, \Delta} m_{\delta, \delta}}\right)
$$

If as a particular case the equality $m_{\Delta, \Delta} m_{\delta, \delta}=0$ holds for graph $G(\Delta, \delta)$, one obtains

$$
n_{\Delta} n_{\delta}=m_{\Delta, \delta}=2 m-\Delta \delta,
$$

from which the main result follows.

Example 1. We present two infinite sequences of balanced bidegreed graphs with the property $m_{\Delta, \Delta} m_{\delta, \delta}=0$. The first infinite sequence is comprised of graphs $B(k)$, where $k$ is a positive integer. The case $k=2$ is depicted in Fig. 5(a). A graph $B(k)$ has a vertex degree distribution $n_{3}=2 k$ and $n_{2 k}=2$, and edge number $m=5 k$, where $k \geq 2$ positive integer. It is easy to see that for graphs $B(k)$, the equality $m_{2 k, 2 k}=0$ holds. 


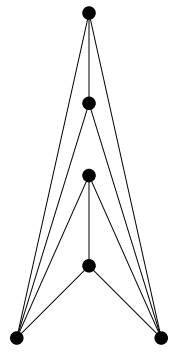

(a)

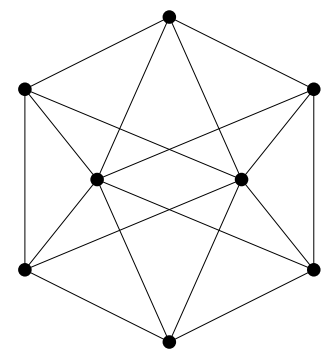

(b)

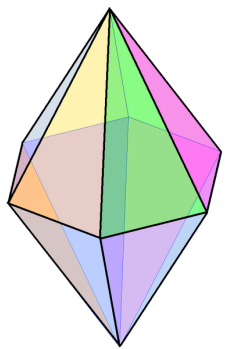

(c)

Fig. 5. Balanced planar bidegreed graphs. (a) Planar graph $B(2)$ and $(b),(c)$ Polyhedral graph $P(6)$ of 6 -gonal bipyramid

The second infinite sequence is comprised of $k$-gonal bipyramids. A $k$-gonal bipyramid, with integer $k \geq 3$, is formed by joining a $k$-gonal pyramid and its mirror image base-to-base. It is a polyhedon having $2 k$ triangular faces. The case $k=6$ is depicted in Fig. $5(b)$ and redrawn in Fig. $5(c)$ for a better illustration. The graph $P(k)$ of a $k$-gonal bipiramid belongs to the family of balanced bidegreed graphs with degree 4 and $k$. For these graphs the equalities $n_{4} n_{k}=m_{4 k}=2 k, m=3 k$ and $m_{k, k}=0$ hold.

\subsection{Complete split graphs and complete split-like graphs}

Proposition 4 ( [2]). There exist a complete split graph pairs with $n$ vertices $G_{c s}(n, q)$ and $G_{c s}(n, q+1)$ with certain $n$ and $q$ positive integers, for which the equality $\operatorname{irr}_{t}\left(G_{c s}(n, q)\right)=\operatorname{irr}_{t}\left(G_{c s}(n, q+1)\right)=\operatorname{irr}\left(G_{c s}(n, q)\right)=\operatorname{irr}\left(G_{c s}(n, q+\right.$ 1)) holds.

Example 2. The smallest complete split graph pair with this property is the star graph on 5 vertices $G_{c s}(5,1)$, and the graph $G_{c s}(5,2)$ are depicted in Fig. 6.

For graphs $G_{c s}(5,1)$ and $G_{c s}(5,2)$ the following equality holds: $\operatorname{irr}_{t}\left(G_{c s}(5,1)\right)=$ $\operatorname{irr}_{t}\left(G_{c s}(5,2)\right)=\operatorname{irr}\left(G_{c s}(5,1)\right)=\operatorname{irr}\left(G_{c s}(5,2)\right)=12$.

Proposition 5. Let $G_{c s l}(n, q, \delta)$ be a complete split-like graph. Then

$$
\operatorname{irr}\left(G_{c s l}(n, q, \delta)\right)=\operatorname{irr}_{t}\left(G_{c s l}(n, q, \delta)\right)=q(n-q)(n-1-\delta) .
$$

Proof. Since the complete split-like graphs form a subset of balanced bidegreed graphs, it is easy to see that

$$
\begin{aligned}
\operatorname{irr}\left(G_{c s l}(n, q, \delta)\right) & =m_{\Delta, \delta}|\Delta-\delta|=n_{\Delta} n_{\delta}|\Delta-\delta|=q(n-q)(n-1-\delta) \\
& =\operatorname{irr}_{t}\left(G_{c s l}(n, q, \delta)\right) .
\end{aligned}
$$




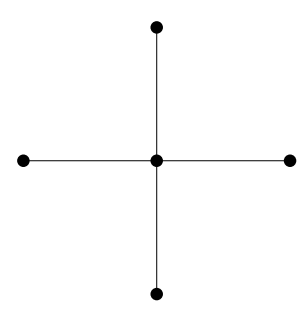

(a)

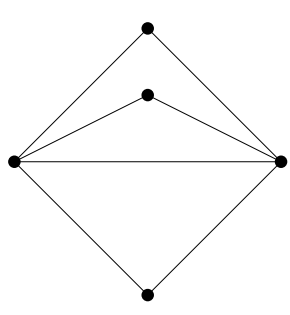

(b)

Fig. 6. Complete split graphs $(a) G_{c s}(5,1)$ and $(b) G_{c s}(5,2)$ with different degree sets

Proposition 6. There exist complete split-like graph pairs $G_{c s l}\left(n_{a}, q_{a}, \delta_{a}\right)$ and $G_{c s l}\left(n_{b}, q_{b}, \delta_{b}\right)$ with different $n_{a}, n_{b}, q_{a}, q_{b}, \delta_{a}$ and $\delta_{b}$ parameters, for which the equality

$$
\begin{aligned}
\operatorname{irr}_{t}\left(G_{c s l}\left(n_{a}, q_{a}, \delta_{a}\right)\right) & =\operatorname{irr}_{t}\left(G_{c s l}\left(n_{b}, q_{b}, \delta_{b}\right)\right)=\operatorname{irr}\left(G_{c s}\left(n_{a}, q_{a}, \delta_{a}\right)\right) \\
& =\operatorname{irr}\left(G_{c s}\left(n_{b}, q_{b}, \delta_{b}\right)\right)
\end{aligned}
$$

holds.

Proof. A complete split-like graph pair with this property is the graph pair $G_{c s l}(5,1,2)$ and $G_{c s l}(6,2,4)$ depicted in Fig. 7. For these graphs, equality $\operatorname{irr}_{t}\left(G_{c s l}(5,1,2)\right)=\operatorname{irr}_{t}\left(G_{c s l}(6,2,4)\right)=\operatorname{irr}\left(G_{c s}(5,1,2)\right)=\operatorname{irr}\left(G_{c s}(6,2,4)\right)=8$ holds.

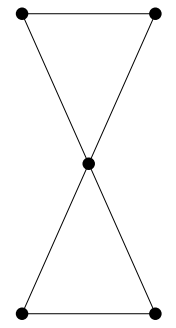

(a)

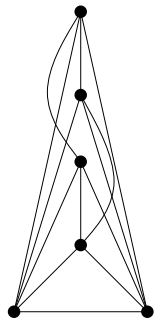

(b)

Fig. 7. Complete split-like graphs $(a) G_{c s l}(5,1,2)$ and $(b) G_{c s l}(6,2,4)$ with equal irr and $\operatorname{irr}_{t}$ measures

There are several ways to construct complete split-like graphs. For example, a complete split-like graph with $n$ vertices $G_{c s l}(n, q, \delta)$ can be generated using the following graph operations:

$$
G_{c s l}(n, q, \delta)=K_{q}+\left(\cup_{j=1}^{J} H(j, R)\right) .
$$


In the formula above, $K_{q}$ is the complete graph on $q \geq 1$ vertices, $H(j, R)$ are $R \geq 1$ regular connected graphs for $j=1,2, \ldots, J$.

As an example, in Fig. 8 two non-isomorphic edge-equivalent complete splitlike graphs are shown. These complete split-like graphs are defined as $G_{c s l}^{1}(14,2,4)=K_{2}+C_{12}$ and $G_{c s l}^{2}(14,2,4)=K_{2}+\left(C_{3} \cup C_{4} \cup C_{5}\right)$, respectively. It is easy to see that $\operatorname{irr}_{t}\left(G_{c s l}^{1}(14,2,4)\right)=\operatorname{irr}_{t}\left(G_{c s l}^{2}(14,2,4)\right)=$

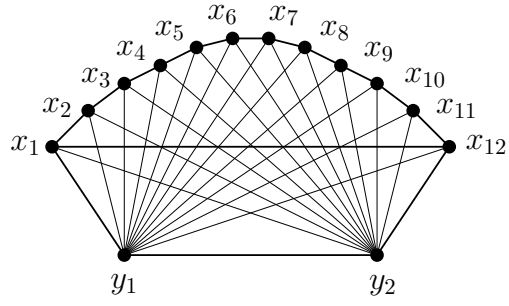

(a)

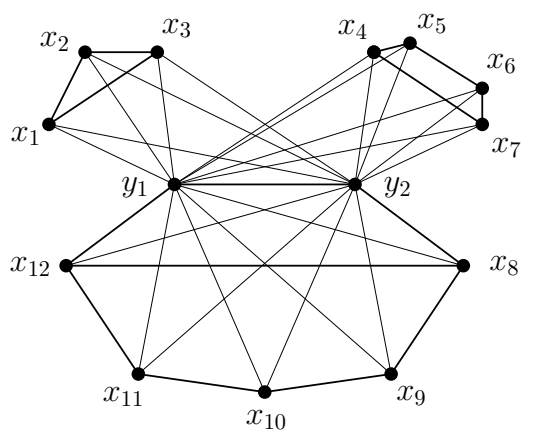

(b)

Fig. 8. Edge-equivalent complete split-like graphs, (a) $G_{c s l}^{1}(14,2,4)$ and (b) $G_{c s l}^{2}(14,2,4)$

$\operatorname{irr}\left(G_{c s l}^{1}(14,2,4)\right)=\operatorname{irr}\left(G_{c s l}^{2}(14,2,4)\right)=216$.

From the previous considerations the following result follows.

Proposition 7. Let $G_{1}$ and $G_{2}$ be edge-equivalent complete split-like graphs. Then the equalities $\operatorname{irr}_{t}\left(G_{1}\right)=\operatorname{irr}_{t}\left(G_{2}\right)=\operatorname{irr}\left(G_{1}\right)=\operatorname{irr}\left(G_{2}\right), \operatorname{Var}\left(G_{1}\right)=$ $\operatorname{Var}\left(G_{2}\right)$ and $\operatorname{CS}\left(G_{1}\right)=\operatorname{CS}\left(G_{2}\right)$ are fulfilled for them.

Proof. Because $G_{1}$ and $G_{2}$ are edge-equivalent graphs, this implies that the equalities $\operatorname{irr}_{t}\left(G_{1}\right)=\operatorname{irr}_{t}\left(G_{2}\right), \operatorname{irr}\left(G_{1}\right)=\operatorname{irr}\left(G_{2}\right)$ and $\operatorname{Var}\left(G_{1}\right)=\operatorname{Var}\left(G_{2}\right)$ hold. Moreover, because $G_{1}$ and $G_{2}$ are complete split-like graphs, in which each vertex is of degree $\delta$ or $n-1$, it follows from Lemma 4 that their spectral radii are identical.

For an illustration of Proposition 7, see the complete split-like graph pair depicted in Fig. 8.

\subsection{Semiregular graphs}

It is important to note that except the complete bidegreed bipartite graphs, the semiregular graphs do not belong to the family of balanced bidegreed graphs. 
Proposition 8. Let $S_{1}\left(\Delta_{1}, \delta_{1}\right)$ and $S_{2}\left(\Delta_{2}, \delta_{2}\right)$ be semiregular graphs for which $\Delta=\Delta_{1}=\Delta_{2}, \delta=\delta_{1}=\delta_{2}$, and $m_{\Delta, \delta}=m\left(S_{1}\right)=m\left(S_{2}\right)$ hold. Then,

$$
\operatorname{CS}\left(S_{1}\right)=\operatorname{CS}\left(S_{2}\right)=\sqrt{\Delta \delta}-\frac{2 \Delta \delta}{\Delta+\delta}
$$

and

$$
\operatorname{Var}\left(S_{i}\right)=\left(\sqrt{\Delta \delta}+\frac{2 \Delta \delta}{\Delta+\delta}\right) \operatorname{CS}\left(S_{i}\right)
$$

for $i=1,2$, where $\operatorname{CS}(G)$ is the Collatz-Sinogowitz irregularity index of a graph $G$.

Proof. It is easy to see that for a semiregular graph $S$ with $n$ vertices

$$
n=n_{\Delta}+n_{\delta}=\frac{m_{\Delta, \delta}}{\Delta}+\frac{m_{\Delta, \delta}}{\delta}=\frac{\Delta+\delta}{\Delta \delta} m_{\Delta, \delta} .
$$

This implies that for the mean degrees $\bar{d}$ we have

$$
\bar{d}\left(S_{1}\right)=\bar{d}\left(S_{2}\right)=\frac{2 m_{\Delta, \delta}}{n}=\frac{2 \Delta \delta}{\Delta+\delta} .
$$

From Lemma 1 one obtains

$$
\rho=\rho\left(S_{1}\right)=\rho\left(S_{2}\right)=\sqrt{\Delta \delta}
$$

consequently, we have

$$
\operatorname{CS}\left(S_{1}\right)=\operatorname{CS}\left(S_{2}\right)=\rho-\frac{2 m_{\Delta, \delta}}{n}=\sqrt{\Delta \delta}-\frac{2 \Delta \delta}{\Delta+\delta} .
$$

Moreover, from Lemmas 2 and 3, it follows that for a semiregular graphs $S$

$$
\begin{aligned}
\operatorname{Var}(S) & =\left(\rho-\frac{2 m}{n}\right)\left(\frac{2 m}{n}+\rho\right)=\rho^{2}-\left(\frac{2 m}{n}\right)^{2}=\Delta \delta-\left(\frac{2 \Delta \delta}{\Delta+\delta}\right)^{2} \\
& =\left(\sqrt{\Delta \delta}+\frac{2 \Delta \delta}{\Delta+\delta}\right) \operatorname{CS}(S) .
\end{aligned}
$$

This implies that

$$
\operatorname{Var}\left(S_{i}\right)=\left(\sqrt{\Delta \delta}+\frac{2 \Delta \delta}{\Delta+\delta}\right) \operatorname{CS}\left(S_{i}\right)
$$

for $i=1,2$.

Proposition 9. Let $S_{1}\left(\Delta_{1}, \delta_{1}\right)$ and $S_{2}\left(\Delta_{2}, \delta_{2}\right)$ be semiregular graphs for which $\Delta=\Delta_{1}=\Delta_{2}, \delta=\delta_{1}=\delta_{2}$, and $m_{\Delta, \delta}=m\left(S_{1}\right)=m\left(S_{2}\right)$ hold. Then, the equalities $\operatorname{irr}_{t}\left(S_{1}\right)=\operatorname{irr}_{t}\left(S_{2}\right), \operatorname{irr}\left(S_{1}\right)=\operatorname{irr}\left(S_{2}\right)$ are fulfilled for them. 
Proof. It is obvious that

$$
\operatorname{irr}\left(S_{1}\right)=\operatorname{irr}\left(S_{2}\right)=m_{\Delta, \delta}(\Delta-\delta) .
$$

Moreover, because for a semiregular graph

$$
n_{\Delta} n_{\delta}=\frac{m_{\Delta, \delta}^{2}}{\Delta \delta},
$$

we get

$$
\operatorname{irr}_{t}\left(S_{1}\right)=\operatorname{irr}_{t}\left(S_{2}\right)=n_{\Delta} n_{\delta}(\Delta-\delta)=\frac{\Delta-\delta}{\Delta \delta} m_{\Delta, \delta}^{2} .
$$

As a consequence of Proposition 8 and 9, we have the following result.

Corollary 2. Let $S_{1}\left(\Delta_{1}, \delta_{1}\right)$ and $S_{2}\left(\Delta_{2}, \delta_{2}\right)$ be semiregular graphs for which $\Delta=\Delta_{1}=\Delta_{2}, \delta=\delta_{1}=\delta_{2}$, and $m_{\Delta, \delta}=m\left(S_{1}\right)=m\left(S_{2}\right)$ hold. Then the equalities $\operatorname{irr}_{t}\left(S_{1}\right)=\operatorname{irr}_{t}\left(S_{2}\right), \operatorname{irr}\left(S_{1}\right)=\operatorname{irr}\left(S_{2}\right), \operatorname{Var}\left(S_{1}\right)=\operatorname{Var}\left(S_{2}\right)$ and $\mathrm{CS}\left(S_{1}\right)=\operatorname{CS}\left(S_{2}\right)$ are fulfilled for them.

Graphs $J_{c}$ and $J_{d}$ depicted in Fig. 4 satisfy Corollary 2. From Proposition 9, we have the following corollary.

Corollary 3. Let $S(\Delta, \delta)$ be a semiregular graph. Then,

$$
\operatorname{irr}_{t}(S(\Delta, \delta))=\frac{\operatorname{irr}^{2}(S(\Delta, \delta))}{\Delta \delta(\Delta-\delta)}
$$

\subsection{Bidegreed graphs with identical CS, Var, irr and $\operatorname{irr}_{t}$ indices}

In Fig. 3(b), Proposition 7 and Corollary 2 examples of pairs of bidegreed graphs were presented, with the property that both graphs from a given pair have identical CS, Var, irr and $\operatorname{irr}_{t}$. Next, we present another such pair of graphs. A 6-vertex graph pair with degree set $\{2,3\}$ and with identical CS, Var, irr and $\operatorname{irr}_{t}$ indices is depicted in Fig. 9. These graphs are edgeequivalent $\left(m_{2,3}=4, m_{3,3}=4\right)$, and they have identical spectral radius $1+\sqrt{3}$.

In the sequel, we show that there exists an infinitely large family of pairs of bidegreed graphs with identical CS, Var, irr and $\operatorname{irr}_{t}$ indices . For that purpose, first we need the following definition:

Let $d_{2}(v)$ denote the sum of the degrees of all vertices adjacent to a vertex $v$ in a graph $G$. Then, $G$ is called 2-walk linear (more precisely, 2-walk $(a, b)$ linear)) if there exists a unique rational numbers pair $(a, b)$ such that

$$
d_{2}(v)=a \cdot d(v)+b
$$

holds for every vertex $v$ of $G$. 

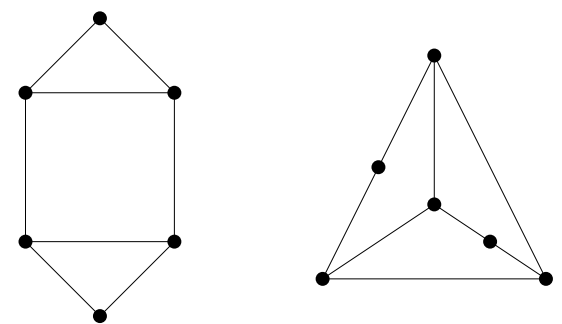

Fig. 9. Tricyclic, bidegreed, edge equivalent graph pair with identical spectral radius $1+\sqrt{3}[9]$

Lemma 5 ( [14]). A graph $G$ has exactly two main eigenvalues if and only if $G$ is 2-walk linear. Moreover, if $G$ is a 2-walk $(a, b)$-linear connected graph, then parameters $a$ and $b$ must be integers, and the spectral radius of $G$ is

$$
\rho=\frac{1}{2}\left(a+\sqrt{a^{2}+4 b}\right) .
$$

Using the above lemma we will demonstrate by examples that there are infinitely many bidegreed graph pairs having identical irregularity indices CS, Var, irr and $\operatorname{irr}_{t}$.

Example 3. Consider the two infinite sequences of bidegreed graphs denoted by $G_{a}(k)$ and $G_{b}(k)$ (an illustration when $k=5$ is given in Fig. 10). Both $G_{a}(k)$ and $G_{b}(k)$ are of order $3 k$, where $k \geq 3$. Graphs $G_{a}(k)$ and $G_{b}(k)$ are

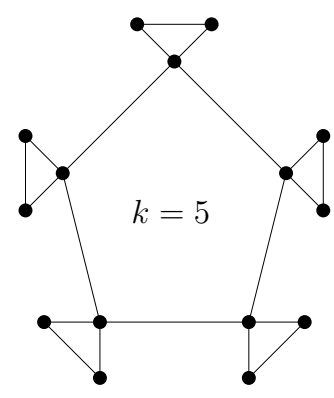

$G_{a}(k)$

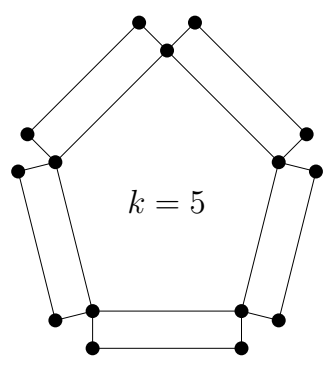

$G_{b}(k)$

Fig. 10. Bidegreed graph pair $G_{a}(5)$ and $G_{b}(5)$

edge-equivalent, because the identities $m_{2,2}=k, m_{2,4}=2 k, m_{4,4}=k, m=4 k$ are fulfilled. Moreover, $G_{a}(k)$ and $G_{b}(k)$ are 2-walk $(3,0)$ linear graphs. By Lemma 5 , it follows that they have identical spectral radius which is equal to 3. It is easy to show that for graphs $G_{a}(k)$ and $G_{b}(k)$ the following equalities hold: $\operatorname{CS}\left(G_{a}(k)\right)=\operatorname{CS}\left(G_{b}(k)\right)=1 / 3, \operatorname{Var}\left(G_{a}(k)\right)=\operatorname{Var}\left(G_{b}(k)\right)=8 / 9$, 
$\operatorname{irr}\left(G_{a}(k)\right)=\operatorname{irr}\left(G_{b}(k)\right)=4 k$, and $\operatorname{irr}_{t}\left(G_{a}(k)\right)=\operatorname{irr}_{t}\left(G_{b}(k)\right)=8 k^{2}$. It is interesting to note that $\operatorname{irr}\left(G_{a}(k)\right) / n=\operatorname{irr}\left(G_{b}(k)\right) / n=4 / 3$, and $\operatorname{irr}_{t}\left(G_{a}(k)\right) / n^{2}$ $=\operatorname{irr}_{t}\left(G_{b}(k)\right) / n^{2}=4 / 9$, for any $k \geq 3$.

Example 4. Another infinite sequence of bidegreed graph pairs denoted by $H_{a}(k)$ and $H_{b}(k)$ is shown in Fig. 11. Each of them has $n=4 k$ vertices, where $k \geq 2$. Graphs $H_{a}(k)$ and $H_{b}(k)$ are edge-equivalent, because the identities

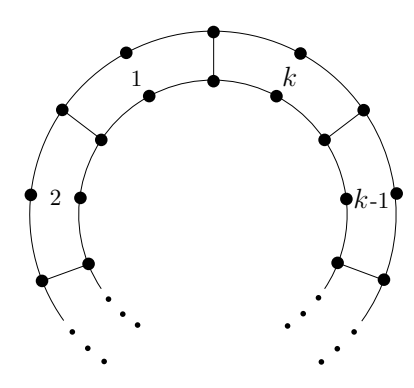

$H_{a}(k)$

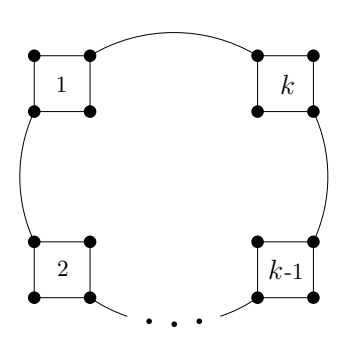

$H_{b}(k)$

Fig. 11. Bidegreed graph pair $H_{a}(k)$ and $H_{b}(k)$

$m_{2,3}=4 k=n, m_{3,3}=k$, and $m=5 k$ hold. It is easy to see that $H_{a}(k)$ and $H_{b}(k)$ are 2-walk $(1,4)$ linear graphs. From this it follows that they have identical spectral radius which is equal to $(1+\sqrt{17}) / 2$. For graphs $H_{a}(k)$ and $H_{b}(k)$ the following equalities hold: $\operatorname{CS}\left(H_{a}(k)\right)=\operatorname{CS}\left(H_{b}(k)\right)=(\sqrt{17}-4) / 2$, $\operatorname{Var}\left(H_{a}(k)\right)=\operatorname{Var}\left(H_{b}(k)\right)=1 / 4, \operatorname{irr}\left(H_{a}(k)\right) / n=\operatorname{irr}\left(H_{b}(k)\right) / n=1$, and $\left.\operatorname{irr}_{t}\left(H_{a}(k)\right) / n^{2}=\operatorname{irr}_{t}\left(H_{b}(k)\right) / n^{2}=1 / 4\right)$.

Example 5. Semi-regular bidegreed graph pairs denoted by $J_{a}(k)$ and $J_{b}(k)$ are shown in Fig. 12. Both of them are comprised of $n=5 k$ vertices, where $k \geq 2$. Graphs $J_{a}(k)$ and $J_{b}(k)$ are edge-equivalent, since the identity $m_{2,3}=$ $6 k$ is fulfilled. Moreover, these graphs are 2-walk $(0,6)$ linear. Consequently, they have identical spectral radius which is equal to $\sqrt{6}$. For graphs $J_{a}(k)$ and $J_{b}(k)$ the following equalities hold: $\operatorname{CS}\left(J_{a}(k)\right)=\operatorname{CS}\left(J_{b}(k)\right)=\sqrt{6}-12 / 5$, $\operatorname{Var}\left(J_{a}(k)\right)=\operatorname{Var}\left(J_{b}(k)\right)=6 / 25, \operatorname{irr}\left(J_{a}(k)\right) / n=\operatorname{irr}\left(J_{b}(k)\right) / n=6 / 5$, and $\operatorname{irr}_{t}\left(J_{a}(k)\right) / n^{2}=\operatorname{irr}_{t}\left(J_{b}(k)\right) / n^{2}=6 / 25$.

\subsection{Smallest bidegreed graphs with identical irregularity indices}

In this section we present pairs of smallest graphs that have identical two or more irregularity measures. The results were obtained by computer search. For two graphs of same order $G_{1}=\left(V_{1}, E_{1}\right)$ and $G_{2}=\left(V_{2}, E_{2}\right)$, we said that $G_{1}$ is smaller than $G_{2}$ if $\left|E_{1}\right|<\left|E_{2}\right|$. Consequently, for two pairs of graphs of same order $D_{1}=\left(G_{1}, G_{2}\right)$ and $D_{2}=\left(G_{3}, G_{4}\right)$, we said that $D_{1}$ is smaller than $D_{2}$ if $\left|E_{1}\right|+\left|E_{2}\right|<\left|E_{3}\right|+\left|E_{4}\right|$. 


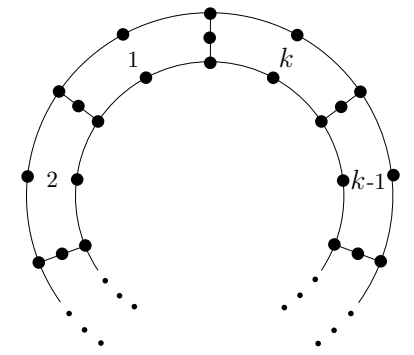

$J_{a}(k)$

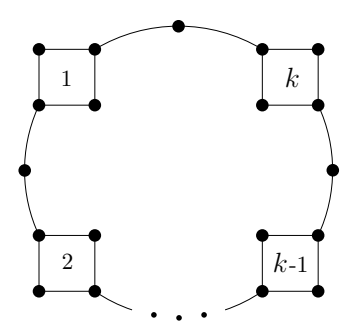

$J_{b}(k)$

Fig. 12. Bidegreed graph pair $J_{a}(k)$ and $J_{b}(k)$

First, in Fig. 13(a) the smallest pair of graphs, that have identical all four irregularity indices CS, Var, irr and $\operatorname{irr}_{t}$, is presented. The graphs $G_{1}$ and $G_{2}$ are of order 6 and size 7 . Their CS, Var, irr and $\operatorname{irr}_{t}$ indices are 0.080880, $0.266667,4$, and 8 , respectively. They also have same spectral radius which is 2.414214 . We note that the pair $\left(G_{1}, G_{2}\right)$ is at same time the smallest pair of graphs with equal CS index.

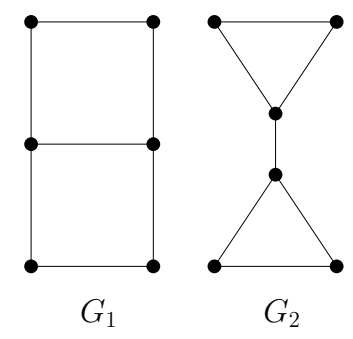

(a)

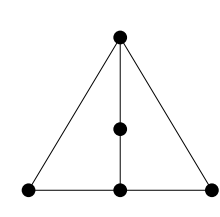

$G_{3}$

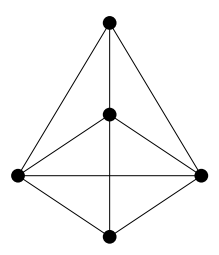

$G_{4}$

$(b)$

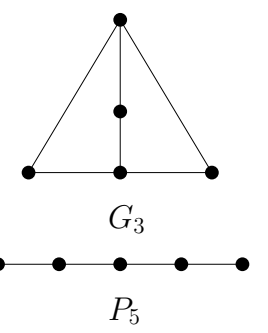

(c)

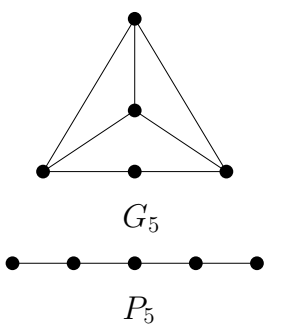

(d)

Fig. 13. Smallest bidegreed graphs with identical irregularity indices 
In Fig. 13(b) the smallest pair of graphs, that have identical Var, irr and $\operatorname{irr}_{t}$ indices is presented. This pair is also the smallest pair with the property that both graphs have equal Var and irr indices. The graphs $G_{3}$ and $G_{4}$ are of order 5 and sizes 6 and 9, respectively. Their Var, irr and irr $_{t}$ indices are $0.300000,6$, and 6 , respectively.

The pair $\left(P_{5}, G_{3}\right)$, depicted in Fig. 13(c), is the smallest pair with the property that both graphs have equal Var and $\operatorname{irr}_{t}$ indices. At same time, it is the smallest pair with both graphs having equal Var index. Also, it is the smallest pair with both graphs having equal $\operatorname{irr}_{t}$ index. Their Var and $\operatorname{irr}_{t}$ indices are 0.300000 and 6 , respectively.

The pair $\left(S_{5}, G_{3}\right)$, depicted in Fig. $13(d)$, is the smallest pair with the property that both graphs have equal irr and $\operatorname{irr}_{t}$ indices. It holds that $\operatorname{irr}\left(S_{5}\right)=$ $\operatorname{irr}\left(G_{3}\right)=12$ and $\operatorname{irr}_{t}\left(S_{5}\right)=\operatorname{irr}\left(G_{3}\right)=12$. At same time, together with the pair $\left(P_{5}, G_{5}\right)$, it is the smallest pair with both graphs having equal irr index.

\section{$5 \quad$ Final remarks and open problems}

In this paper we focused our investigation to the study of the relations between the irregularity indices of bidegreed connected graphs. Comparing the irregularity indices of various graphs, in the majority of cases it was supposed that the number of vertices or the corresponding degree sets are identical (see Figures $3,4,6,8,9,10,11,12,13(a))$. It would be interesting to consider graphs of same order which have different degree sets, but their corresponding irregularity indices are identical (as few examples in Fig. 13(b), $(c),(d)$ ).

Another interesting problem is to estimate the maximum possible difference of vertex and edge numbers of graphs having identical irregularity indices (assuming that such positive finite integer exists.) Both cases, when graphs are of same or different order, are of interest. In Fig. 14, bidegreed graphs $B(6,5)$ and $B(3,2)$ represent an example concerning this problem. We would

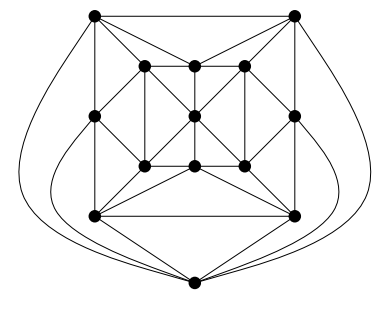

$$
B(6,5)
$$

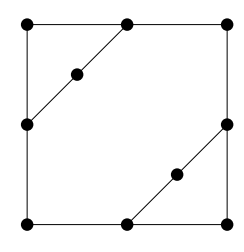

$B(3,2)$

Fig. 14. Bidegreed graphs with identical $\operatorname{irr}_{t}=24$ and irr $=12$ indices

like to note that, the bidegreed polyhedral graph $B(6,5)$ is the dual of the 
graph of the smallest $C_{24}$ fullerene which is composed of 12 pentagonal and 2 hexagonal faces, and graph $B(3,2)$ is a semiregular graph. It is worth noting that graph $B(6,5)$ has 14 vertices and 36 edges, while graph $B(3,2)$ has 10 vertices and 12 edges. It is surprising that there is a large difference between the corresponding edge-numbers of the two graphs, $(36-12=24)$.

\section{References}

1. H. Abdo, D. Dimitrov, The total irregularity of a graph, arxiv.org/abs/1207. 5267, 2012.

2. H. Abdo, N. Cohen, D. Dimitrov, Bounds and computation of irregularity of a graph, http://arxiv.org/abs/1207.4804, 2012.

3. Y. Alavi, J. Liu, J. Wang, Highly irregular digraphs, Discrete Math. 111 (1993) $3-10$.

4. M. O. Albertson, The irregularity of a graph, Ars Comb. 46 (1997) 219-225.

5. M. Aouchiche, F. K. Bell, D. Cvetković, P. Hansen, P. Rowlinson, S. K. Simić, D. Stevanović, Variable neighborhood search for extrenmal graphs. 16. Some conjectures related to the largest eivenvalue of a graph, Eur. J. Oper. Res. 191 (2008) 661-676.

6. F. K. Bell, On the maximal index of connected graphs, Linear Algebra Appl. 144 (1991) 135-151.

7. F. K. Bell, A note on the irregularity of graphs, Linear Algebra Appl. 161 (1992) 45-54.

8. L. Collatz, U. Sinogowitz, Spektren endlicher Graphen, Abh. Math. Sem. Univ. Hamburg 21 (1957) 63-77.

9. X. Fan, Y. Luo, Tricyclic graphs with exactly two main eigenvalues, http://arxiv.org/abs/1012.0963, 2010.

10. I. Gutman, P. Hansen, H. Mélot, Variable neighborhood search for extremal graphs. 10. Comparison of irregularity indices for chemical trees, J. Chem. Inf. Model. 45 (2005) 222-230.

11. P. Hansen, H. Mélot, Variable neighborhood search for extremal graphs. 9. Bounding the irregularity of a graph, in Graphs and Discovery, DIMACS Ser. Discrete Math. Theoret. Comput. Sci 69 (2005) 253-264.

12. M. A. Henning, D. Rautenbach, On the irregularity of bipartite graphs, Discrete Math. 307 (2007) 1467-1472.

13. Y. Hong, J-L. Shu, K. Fang, A sharp upper bound of the spectral radius of graphs, J. Combin. Theory Ser. B 81 (2001) 177-183.

14. Y. Hou, F. Tian, Unicyclic graphs with exactly two main eigenvalues, Appl. Math. Lett. 19 (2006) 1143-1147.

15. P. Rowlinson, The main eigenvalues of a graph: A survey, Appl. Anal. Discrete Math. 1 (2007) 445-471.

16. A. Yu, M. Lu, F. Tian, On the spectral radius of graphs, Linear Algebra Appl. 387 (2004) 41-49. 